\title{
Synthetic spectra of A supergiants
}

\author{
D. Korčáková ${ }^{1}$, J. Kubát ${ }^{1}$, J. Krtička ${ }^{2}$ and M. Šlechta ${ }^{1}$ \\ ${ }^{1}$ Astronomical Institute, Academy of Sciences of the Czech Republic, Fričova 298, CZ-251 65 \\ Ondřejov, Czech Republic \\ email: kor@sunstel.asu.cas.cz, kubat@sunstel.asu.cas.cz, slechta@sunstel.asu.cas.cz \\ ${ }^{2}$ Institute of Theoretical Physics and Astrophysics, Masaryk University, Kotrlářská 2, \\ CZ-611 37 Brno, Czech Republic \\ email: krticka@physics.muni.cz
}

\begin{abstract}
The stellar winds of A supergiants can have a significant influence on their emergent spectra. Here we present the hydrogen line profiles of a model based on the stellar parameters of HD 12953. The radiative transfer equation is solved in two dimensions in axial symmetry. We do not include the velocity field by the Sobolev approximation, but in detail using the Lorentz transformation. This allows us to correctly include the stellar wind, since the velocity gradients in A supergiants are too small for the Sobolev approximation to be valid.
\end{abstract}

Keywords. Hydrodynamics, line: profiles, radiative transfer, (stars:) supergiants, stars: individual (HD 12953)

\section{Introduction}

A supergiants are slowly rotating stars with stellar winds. Their equatorial rotational velocities are usually less than $50 \mathrm{~km} \mathrm{~s}^{-1}$. Due to these stellar winds their strong lines show P Cygni profiles and the weak lines are asymmetric. The stellar spectra as well as the photometry show variability. The first synthetic spectra of A supergiants were calculated by Wolf $(1971,1972)$ and Parsons \& Peytremann (1973). They assumed planeparallel geometry and the atmosphere in hydrostatic equilibrium and in LTE but did not include line blanketing. Later ATLAS9 (Kurucz 1993) models, which include this effect (e.g., Verdugo et al. 1999) were used. A better approximation was made by Przybilla (2002), who used LTE model atmospheres and calculated the emergent spectra from the solution of the equations of statistical equilibrium. The first attempt to solve the wind of A supergiant together with photospheric layers has been done by Aufdenberg et al. (2002). Since A supergiants have stellar winds with a very low velocity gradient, the condition for Sobolev approximation is not fulfilled there. We present a method for solving the radiative transfer equation that is appropriate for this case.

For our results the influence of the velocity field on the spectral lines of the star is modeled. The parameters correspond to those of HD 12953 (HR 618), an A supergiant with an effective temperature of $9100 \mathrm{~K}$, a radius of $145 R_{\odot}$ and a mass of about $9.7 M_{\odot}$ (Kudritzki et al. 1999).

\section{Description of models}

Our calculations are based on two numerical models. The state parameters, electron density and temperature, are obtained using the hydrostatic spherically symmetric model atmosphere code ATA (see Kubát 2003). These parameters serve as the input data to the $2 \mathrm{D}$ radiative transfer code (Korčáková 2003), which solves the equation of radiative transfer. 


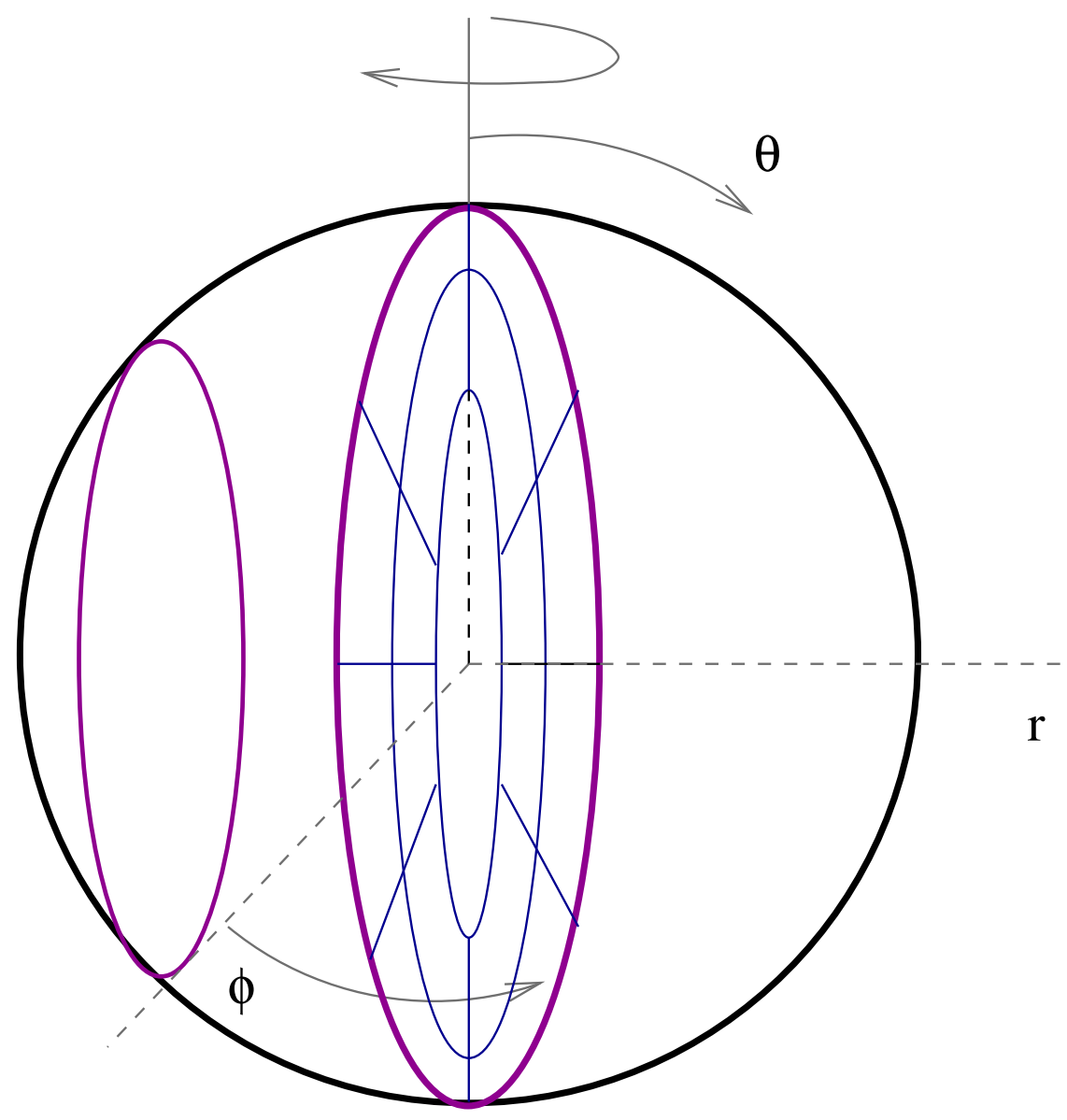

Figure 1. The scheme of the set of longitudinal planes.

\subsection{Hydrostatic code}

The code calculates spherically symmetric static NLTE model atmospheres. It solves the equations of hydrostatic, radiative, and statistical equilibrium. The radiation field is accounted for using the method of approximate lambda operators. During the formal solution step the static spherically symmetric radiative transfer equation is solved using Feautrier variables. The temperature structure is calculated with the help of the electron thermal balance method (Kubát et al. 1999). Although the code is able to include an arbitrary number of chemical elements, for simplicity, we used a pure hydrogen model here.

\subsection{Solution of the radiative transfer equation}

We assume axial symmetry for the solution of the radiative transfer equation. The transfer problem is solved independently in longitudinal planes, which intersect the star (Fig. 1). The whole radiation field is obtained by rotating these planes around the axis of symmetry (Fig. 2). In each plane the transfer problem is solved using a combination of the short and long characteristic method (Fig. 3).

The ray starts and ends at the grid circle (Fig. 3), so it is possible to intersect more cells. This better allows us to include the global character of the radiation field. The 


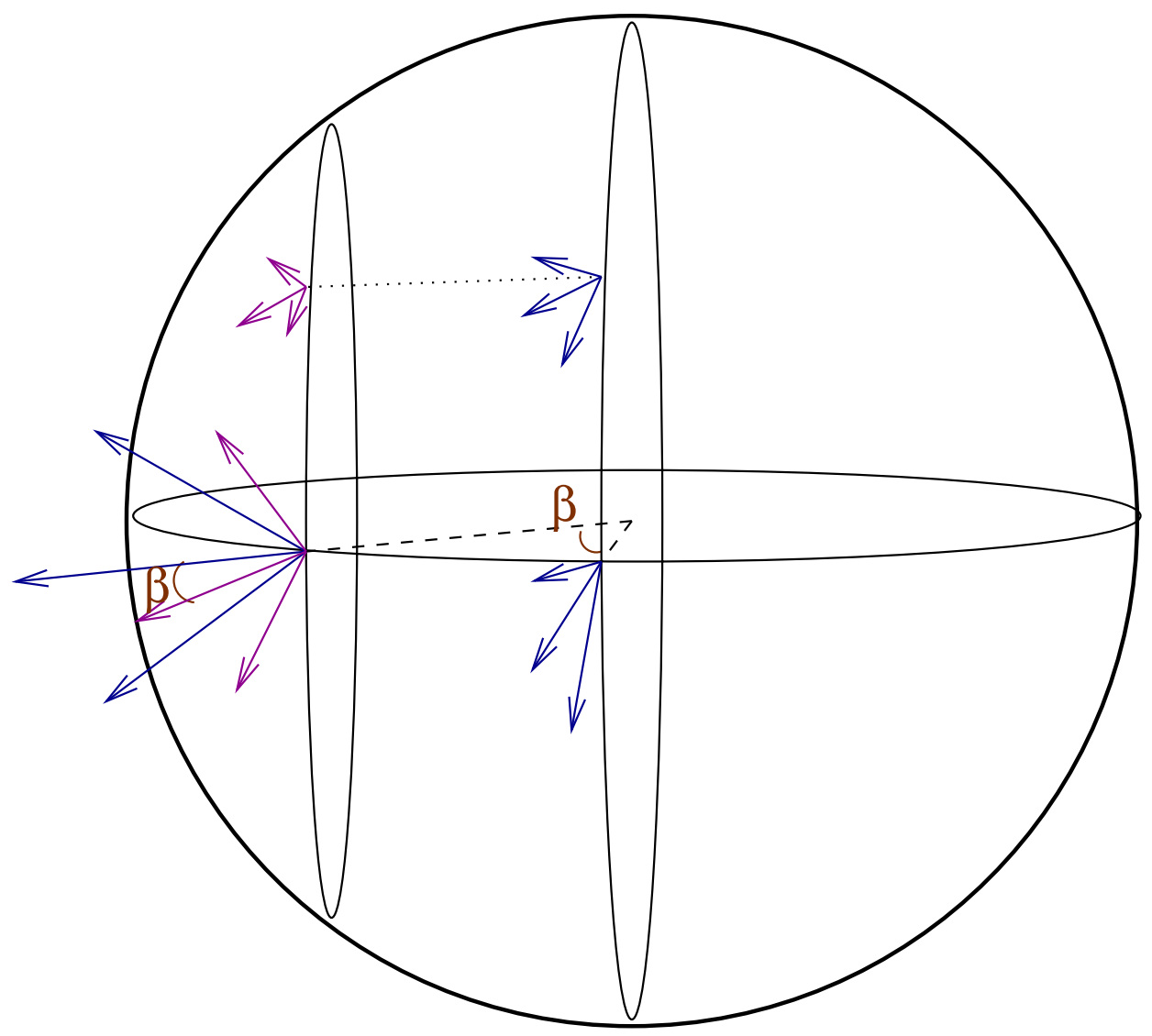

Figure 2. The scheme for calculating the whole radiation field.

transfer equation is then calculated along selected rays

$$
I_{(B)}=I_{(A)} e^{-\Delta \tau_{(A B)}}+\int_{0}^{\Delta \tau_{(A B)}} S(t) e^{\left[-\left(\Delta \tau_{(A B)}-t\right)\right]} d t .
$$

The interval $A B$ is a section of the ray, which is in one cell. We assume the source function as well as the opacity to change linearly within this interval.

In every cell we assume a constant velocity and its change is permitted only at the boundary of cells. This approximation allows us to solve the static equation of radiative transfer in the cells. At the cell boundaries we perform the transformation of frequency (it is possible to neglect the transformation of intensity in this case due to the small velocity gradient). A more detailed description of the method will be published elsewhere (Korčáková \& Kubát 2004).

\section{Results}

The aim of this paper is to show the ability to calculate synthetic spectra of an expanding A supergiant atmosphere. We consider the spherical symmetry, although it is not necessary for the radiative transfer code. Since we do not have a consistent radiative hydrodynamics model of the wind, we used as input a model derived from the hydrostatic code by multiplying the radius scaled by $r^{\text {new }}(d)=d^{1.16} r^{\text {old }}(d)$, where $d=1 \ldots N D$ is the index of the depth point, to obtain an observable $\mathrm{P}$ Cygni profile. We adopt the $\beta$ 


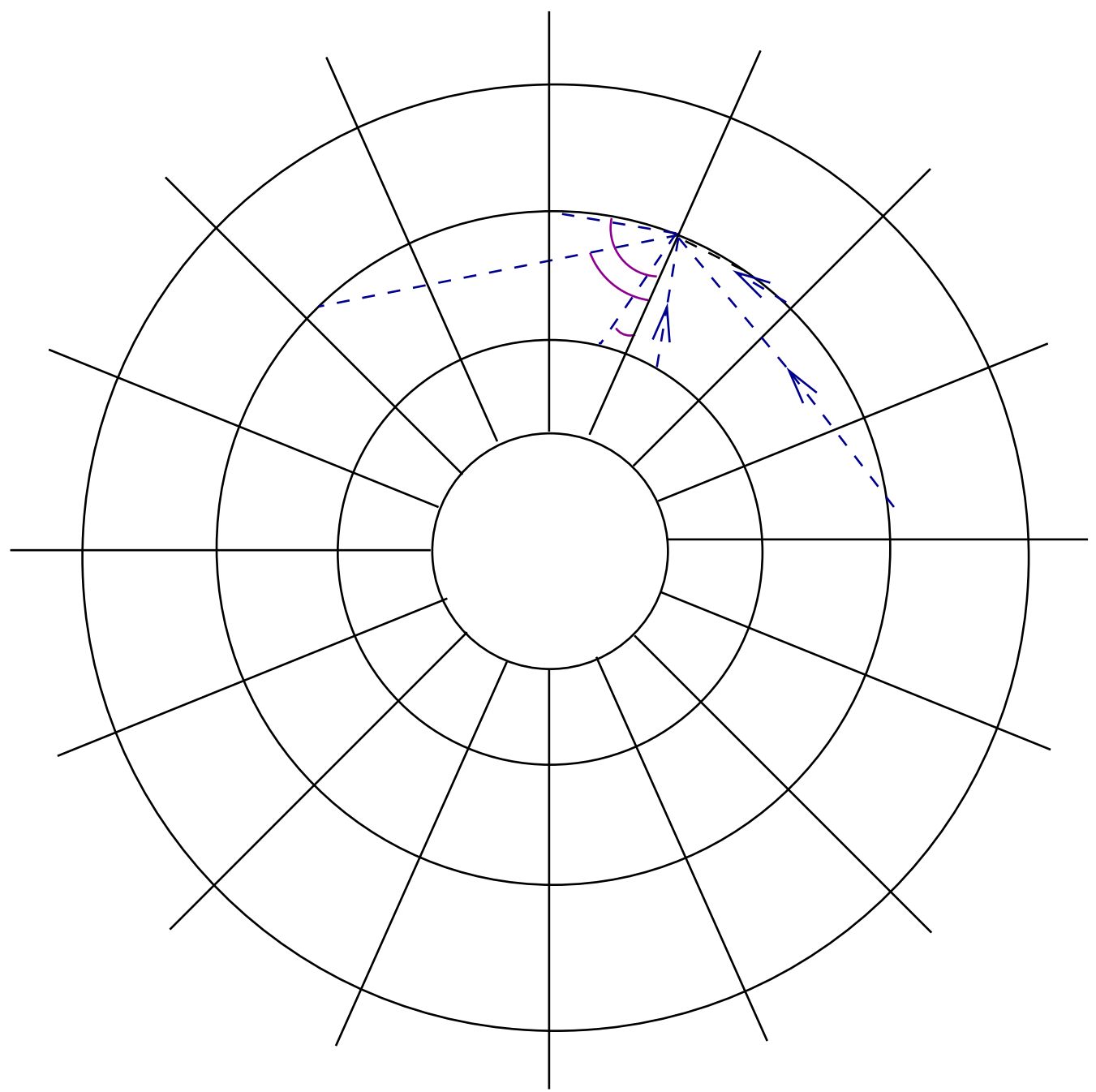

Figure 3. The scheme for solving the radiative transfer in the longitudinal plane.

velocity law (see, e.g., Cassinelli \& Lamers 1999)

$$
v(r)=v_{\infty}\left\{1-\left[1-\left(\frac{v_{R}}{v_{\infty}}\right)^{\frac{1}{\beta}}\right] \frac{R}{r}\right\}^{\beta},
$$

with parameter $\beta=1$ and the terminal velocity $v_{\infty}=280 \mathrm{~km} \mathrm{~s}^{-1}$ for the velocity field. We choose the velocity in the photosphere $v_{R}=0.2 \mathrm{~km} \mathrm{~s}^{-1}$. This is not a physically consistent model. However, we want to present the new method for solving radiative transfer. Currently we are working to couple this code with the hydrodynamic one described in Krtička \& Kubát (2004).

In Fig. 4 the $\mathrm{H} \alpha$ line profile obtained from our code is plotted. This line shows the $\mathrm{P}$ Cygni profile, which is observed in HD 12953. Since we do not take the input data from the hydrodynamic model, and we do not include NLTE effects, our line is weaker than in the observed spectrum (where the relative intensity in emission is 1.62). Note that the system of equations of the statistical equilibrium is included in our code. However, even 


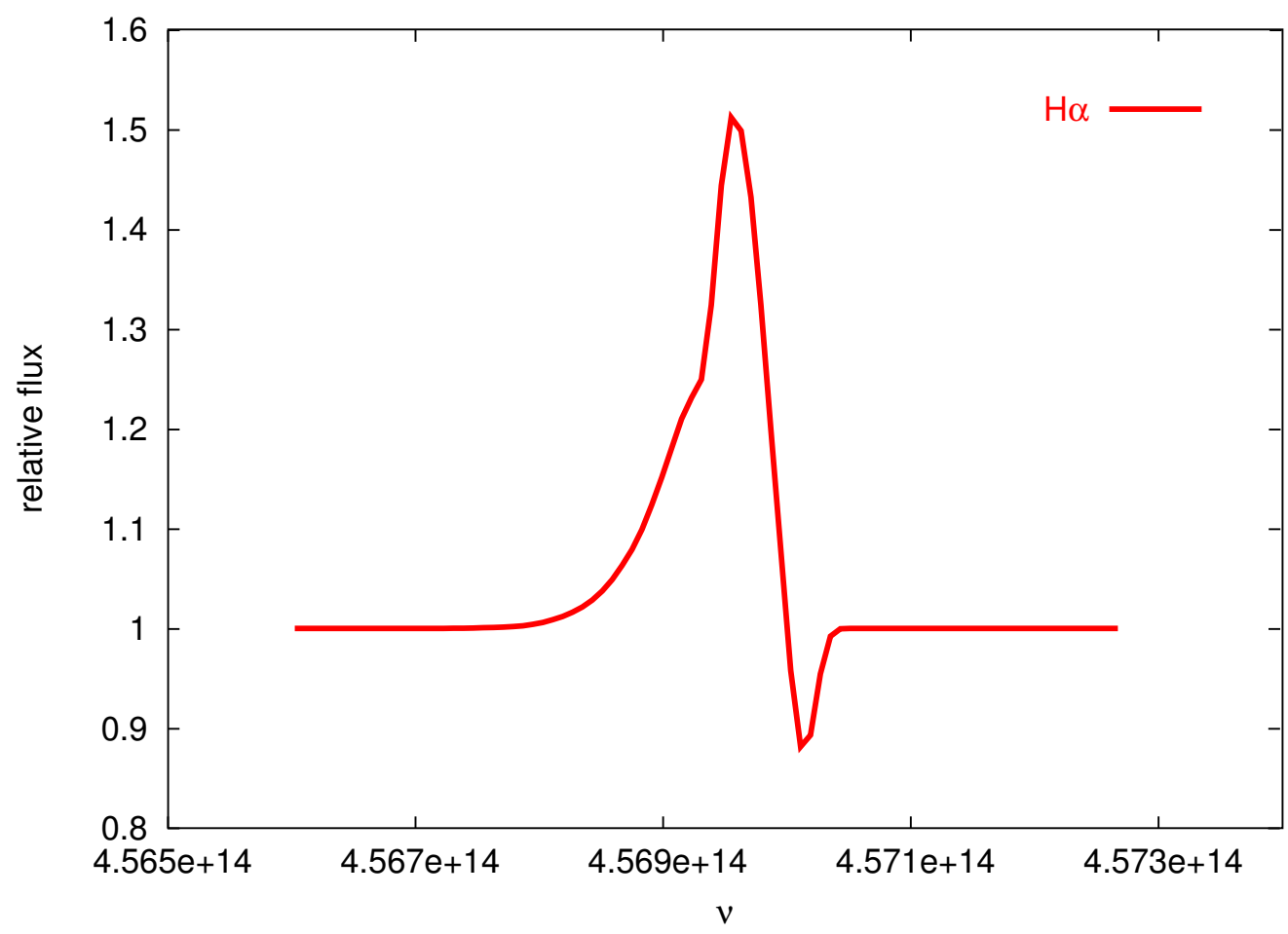

Figure 4. The $\mathrm{H} \alpha$ line profile calculated using the $2 \mathrm{D}$ radiative transfer code.

a pure formal solution in the $2 \mathrm{D}$ case with velocity fields is very time consuming. Thus for demonstration purposes we preferred the faster LTE variant to save some computing time.

In Fig. 5 we show the limb darkening. The emission comes from the far extended region. Note, if we want to describe stellar rotation in such an extended atmosphere, we have to use a more accurate method than the simple convolution of static and rotation profiles. Failing to do this we will obtain the wrong value of the rotational velocity. Our code is able to solve the transfer problem in rapidly rotating stars as well.

\section{Conclusion}

We present here a new method for solving the radiative transfer equation in axial symmetry which includes the velocity field. Our method considers a constant value of velocity in the cells and permits changes of velocity only at the cell boundaries. This approximation allows us to include the small velocity gradients as well as the large (but not relativistic) ones in the stellar winds of hot stars. The calculated $\mathrm{H} \alpha$ line profile of the P Cygni type is plotted in Fig. 4.

Our code is appropriate for calculating flux in the lines formed in rotating winds. Since these stars have extended atmospheres, the emission region is too large for the approximation of radiative transfer using the plane-parallel model. However a full 3D calculation is not necessary. This code is also applicable to a solution of the radiative transfer equation in accretion discs (Korčáková et al. 2004). In this case, it is possible to include not only the radiation from the central object and the disk itself, but also from the hot corona and the wind from the inner parts of the disk. 


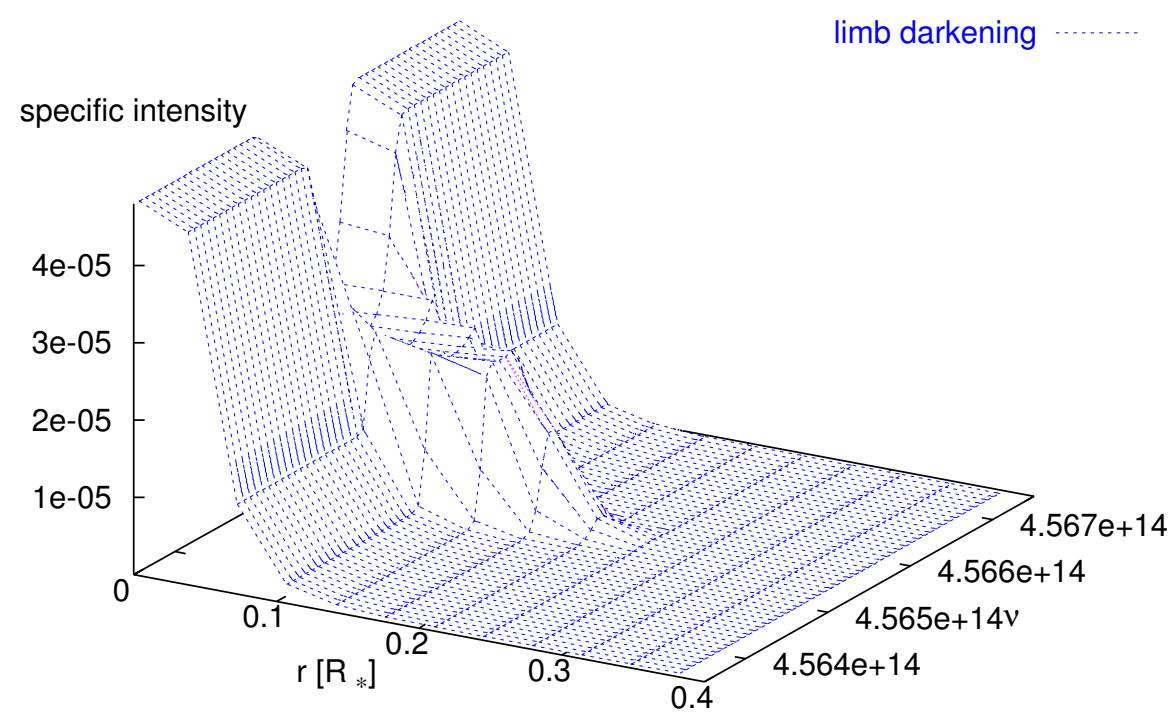

Figure 5. Limb darkening in the $\mathrm{H} \alpha$ line.

\section{Acknowledgements}

This research has made use of NASA's Astrophysics Data System. This work was supported by grants GA ČR 205/02/0445, 205/04/1267, and 205/04/P224. The Astronomical Institute Ondřejov is supported by projects K2043105 and Z1003909.

\section{References}

Aufdenberg, J. P., Hauschildt, P. H., Baron, E., Nordgren, T. E., Burnley, A. W., Howarth, I. D., Gordon, K. D., Stansberry, J. A., 2002, ApJ, 570, 344

Cassinelli, J. P., \& Lamers, H. J. G. L. M., 1999, Introduction to Stellar Winds, Cambridge Univ. Press, Cambridge

Korčáková, D. 2003, PhD thesis, Masaryk University Brno

Korčáková, D. \& Kubát, J. 2004, A\&\&A, submitted

Korčáková, D., Kubát, J., Kawka, A. 2004, in D. Koester \& S. Moehler (eds.), 14th European Workshop on White Dwarfs, Astron. Soc. Pacific Conf. Ser., in press

Krtička, J. \& Kubát, J. 2004, A\&\&A 417, 1003

Kubát, J., 2003, in N. E. Piskunov, W. W. Weiss \& D. F. Gray (eds.), Modelling of Stellar Atmospheres, IAU Symp. 210, Astron. Soc. Pacific, A8

Kubát, J., Puls, J., Pauldrach, A. W. A. 1999, A\&\& A 341, 587

Kudritzki, R. P., Puls, J., Lennon, D. J., Venn, K. A., Reetz, J., Najarro, F., McCarthy, J. K., Herrero, A., 1999, ApJ 350, 970

Kurucz, R. L. 1993, Solar Abundance Model Atmospheres, Kurucz CD-ROM No.19

Parsons, S. B. \& Peytremann, E. 1973, ApJ 180, 71

Przybilla, N. 2002, PhD thesis, Ludwig-Maximilians-Universität München

Venn, K. A. 1995, ApJS 99, 659

Verdugo, E., Talavera, A., Gómez de Castro, A. I. 1999, A\&\& 346, 819

Wolf, B. 1971, A\&A 10, 383

Wolf, B. 1972, A\&B 20, 275 